\title{
Revision of Perineal Urethrostomy Using a Meshed Split-Thickness Skin Graft
}

\author{
N. Lumen ${ }^{a} \quad$ P. Houtmeyers ${ }^{b} \quad$ S. Monstrey ${ }^{b} \quad$ A.-F. Spinoit ${ }^{a}$ \\ W. Oosterlinck ${ }^{\mathrm{a}}$ P. Hoebeke ${ }^{\mathrm{a}}$ \\ Departments of ${ }^{a}$ Urology and ${ }^{b}$ Plastic and Reconstructive Surgery, Ghent University \\ Hospital, Ghent, Belgium
}

\section{Key Words}

Urethral stricture · Perineostomy · Perineal urethrostomy · Urethroplasty

\begin{abstract}
Perineal urethrostomy is considered to be the last option to restore voiding in complex/recurrent urethral stricture disease. It is also a necessary procedure after penectomy or urethrectomy. Stenosis of the perineal urethrostomy has been reported in up to $30 \%$ of cases. There is no consensus on how to treat a stenotic perineal urethrostomy, but, in general, a form of urinary diversion is offered to the patient. We present the case of a young male who underwent perineal urethrostomy after urethrectomy for urethral cancer. The postoperative period was complicated by wound dehiscence with subsequent complete obliteration of the perineal urethrostomy. Revision surgery was performed with reopening of the obliterated urethral stump and coverage of the skin defect between the urethra and the perineal/scrotal skin with a meshed split-thickness skin graft. To date, this patient is voiding well and satisfied with the offered solution.

(C) 2014 S. Karger AG, Basel
\end{abstract}

\section{Introduction}

Perineal urethrostomy is an option for the reconstructive urologist for complex and/or refractory urethral strictures [1-3]. It is generally considered as the last option before abandoning the urethral outlet. Furthermore, perineal urethrostomy is a necessity after penectomy for penile cancer or urethrectomy for urethral cancer $[4,5]$. Unfortunately, stenosis of the perineal urethrostomy is possible and reported in up to $30 \%$ of cases $[1-3,6]$. In these cases, the urethral outlet is often abandoned and some form of urinary diversion is

Prof. Dr. Nicolaas Lumen, MD, PhD

Department of Urology

Ghent University Hospital

De Pintelaan 185, BE-9000 Ghent (Belgium)

E-Mail Nicolaas.lumen@uzgent.be 
offered (e.g. suprapubic catheter or appendicovesicostomy). Literature on revision of the stenotic perineal urethrostomy is extremely scarce. The use of a meshed split-thickness skin graft in urethral surgery was introduced by Schreiter and Noll [7]. They used this graft as a substitute in two-stage urethroplasty. We thought this graft might be useful in the revision of a stenotic perineal urethrostomy.

\section{Case Report}

A 45-year-old male patient underwent partial penectomy and distal urethrostomy for erythroplasia of Queyrat (i.e. carcinoma in situ of squamous cell carcinoma) in 2008. Since he was extremely dissatisfied with the remaining penile stump, he consulted our center about the option of phallic reconstruction. On June 14, 2010, phallic reconstruction was performed. The native urethra was lengthened by means of a radial forearm free flap. The phallus was reconstructed with an anterolateral thigh flap. The urethra was positioned inside the phallus according to the 'tube-in-a-tube' principle. In October 2012, the patient presented with obstructive voiding symptoms. A diagnostic urethroscopy showed a suspicious lesion in the remaining native, bulbar urethra, and biopsy confirmed the recurrence of a poorly differentiated squamous cell carcinoma of the urethra. On December 13,2012 , he underwent excision of the remaining native, bulbar urethra with the creation of a perineal urethrostomy at the membranous urethra. The perineal urethrostomy was performed using the technique as described by Blandy et al. [8]. Unfortunately, the postoperative period was complicated by bleeding, wound dehiscence and infection (fig. 1). Finally, the perineal urethrostomy became completely obliterated and a suprapubic catheter was placed (fig. 1). As the patient accepted this only as a temporary solution, we proposed the revision of the perineal urethrostomy using a meshed split-thickness skin graft. This procedure was performed on April 23, 2013. The patient was placed in the lithotomy position and a midline perineal incision was made. Dissection was continued up to the level of the urogenital diaphragm. A flexible cystoscope was inserted along the suprapubic tract and brought through the bladder neck into the posterior urethra. This marked the place of obliteration as the light of the cystoscope could be seen through the perineal incision. The urethra was opened on the tip of the cystoscope. All fibrotic tissue was further excised until only healthy urethral mucosa remained. The urethral stump was spatulated over $1.5 \mathrm{~cm}$. Vicryl 4.0 stay sutures were placed at the mucosa to facilitate identification and to prepare the anastomosis for the graft (fig. 2). A split-thickness graft $(10 \times 15 \mathrm{~cm})$ was harvested from the skin of the left thigh and meshed 1:1. In the middle of the graft, an opening was made to anastomose with the urethral opening. The edges of the graft were further tailored and anastomosed with the perineal skin edges. Several additional quilting sutures were placed to fix the graft against the perineal graft bed (fig. 3). A compressive dressing and a $20-\mathrm{Fr}$ urethral catheter were left in place for 5 days. After 5 days, the dressing was removed with satisfactory graft take. Subsequently, the catheter was also removed. At present ( 6 months of follow-up), the patient is voiding well. Maximum and average urinary flow are 22 and 13 $\mathrm{ml} / \mathrm{s}$, respectively. His International Prostate Symptom score is 5/35. The perineal wound has healed well, with minimal contraction of the grafted area (fig. 3). Through the neomeatus, a 20-Fr catheter can be passed without any difficulties, indicating a sufficient diameter.

This study was approved of by the local ethics committee. 
Lumen et al.: Revision of Perineal Urethrostomy Using a Meshed Split-Thickness Skin Graft

\section{Discussion}

Perineal urethrostomy is often considered the last option to restore voiding before abandoning the urethral outlet in complex urethral stricture disease [9]. Perineal urethrostomy is also needed after penectomy or urethrectomy for penile and urethral cancer, respectively $[4,5]$. In this case, it was necessary to perform a perineal urethrostomy after resection of the bulbar urethra because of urethral squamous cell carcinoma. This is the second most important type of urethral malignancy [4]. For perineal urethrostomy, several surgical techniques have been described (7-flap [1], Blandy et al. [8] and Johanson [10]). These techniques are based on the use of local perineal and/or scrotal skin flaps that are sutured to the opened proximal urethra.

Nevertheless, success is far from guaranteed since stenosis of the perineal urethrostomy is reported in $10-30 \%$ of cases $[1-3,6]$. Several reasons for this are possible. (1) It might be recurrence of lichen sclerosus at the neomeatus if lichen sclerosus was the initial etiology of the urethral stricture [9]. (2) The quality of the urethra where the perineal urethrostomy is performed might be poor (especially after previous stricture repair, perineal trauma or irradiation) and this might lead to further scarring and narrowing [2, 3]. (3) There may be tension between the anastomosis of the urethra and the local skin flaps, with wound dehiscence and excessive scar tissue formation. It might be difficult to bring local skin flaps towards the urethra without any tension, especially when a perineal urethrostomy has to be performed at the level of the membranous or deep bulbar urethra.

The latter is obviously the reason for failure of the perineal urethrostomy in this case report. No consensus exists on how to manage the stenotic perineal urethrostomy and literature on this issue is extremely scarce. Several options have been suggested and are outlined below.

First, the urethral outlet is abandoned and a permanent suprapubic catheter is inserted. However, a permanent suprapubic catheter is often not accepted by patients, especially younger ones. The patient in this case definitely refused this option. Furthermore, a permanent suprapubic catheter is associated with significant complications such as recurrent urinary tract infection and the formation of bladder stones.

Second, the urethral outlet is abandoned and some form of urinary derivation is performed. In case of a normal bladder capacity, a continent catheterizable diversion is preferred (Mitrofanoff or Monti procedure). This is already major surgery requiring the opening of the peritoneal cavity. If the appendix is not suitable or was previously removed, an ileal segment can be used. In this case, an intestinal anastomosis has to be performed with its possible complications. Intermittent catheterization of the stoma requires dexterity and compliance of the patient. Furthermore, stomal stenosis requiring corrective surgery has been reported [11]. The patient in this case also refused this option.

Third, dilation of the stenotic neomeatus may be performed, and, if necessary, the patient can be taught to perform intermittent self-dilation [1,2]. This was not possible in this patient because of the complete obliteration of the neomeatus and the absence of a tract that could be dilated.

Fourth, a repeat perineal urethrostomy with local skin flaps (perineal or scrotal) [2, 3] may be done. For example, a Johanson perineal urethrostomy can be performed after failure of a Blandy perineostomy or vice versa. Paucity of local skin hampered this option in our patient. Indeed, the urethral stump was situated deep in the perineal incision and there was already tension between the local skin flaps and the urethra at the time of initial perineal urethrostomy, finally leading to dehiscence and failure. 
Finally, revision of the perineal urethrostomy is also an option. Kamat [9] has reported the use of buccal mucosa to augment the stenotic neomeatus. We consider this technique as a valuable option in case of a short stenosis of the neomeatus that is not completely obliterated. The main problem in our patient was a large defect between the opened urethral stump at the level of the urogenital diaphragm and the perineal skin. This extensive defect could not be covered with buccal mucosa. A meshed split-thickness skin graft is an option as a substitute in urethral reconstruction when buccal mucosa is no longer available or contraindicated $[7,12-14]$. A success rate of $80-93 \%$ has been reported [12-15]. We thought it might be useful in this case to fill up the defect between the opened urethral stump and the local perineal and scrotal skin. This option was offered to and accepted by the patient.

Important advantages of a meshed split-thickness skin graft are its large availability, it is non-hair bearing and easy to tailor into the defect. The main disadvantage of this graft is its tendency to retract $[13,14]$. We therefore advise broad spatulation of the urethral stump in order to compensate for circumferential retraction at the neomeatus. Some minor retraction of the graft elsewhere is not a problem since it will not be used for further reconstruction in this patient with a definitive perineostomy. As with all grafts, it is of utmost importance to place the graft onto a well-vascularized graft bed. Therefore, we performed resection of all fibrotic tissue surrounding the urethral stump to assure satisfactory graft take. This is also important to avoid graft retraction.

To our knowledge, this is the first report of the use of a meshed split-thickness skin graft in the revision of a stenotic perineal urethrostomy. This technique is very similar to the first stage of a two-stage urethroplasty with a mesh graft $[7,13,14]$; however, in this case the mesh graft was used to fill up the perineal skin defect rather than to substitute the urethra. Further research is needed with more patients and longer follow-up to define the true value of this technique. At the moment, we offer this option to all our patients with a stenotic perineal urethrostomy, and this is now the subject of a prospective study also considering issues related to quality of life.

\section{Conclusion}

Stenosis of the perineal urethrostomy is a major challenge for the reconstructive urologist. Revision of the perineal urethrostomy and augmentation of the neomeatus with the use of a meshed split-thickness skin graft is a feasible option to restore urethral voiding. Further research is needed to establish the true value of this technique.

\section{References}

1 French D, Hudak SJ, Morey AF: The '7-flap' perineal urethrostomy. Urology 2011;77:1487-1489.

2 Myers JB, Porten SP, McAninch JW: The outcomes of perineal urethrostomy with preservation of the dorsal urethral plate and urethral blood supply. Urology 2011;77:1223-1227.

3 Barbagli G, De Angelis M, Romano G, Lazzeri M: Clinical outcome and quality of life assessment in patients treated with perineal urethrostomy for anterior urethral stricture disease. J Urol 2009;182:548-557.

4 Gakis G, Witjes JA, Comperat E, et al: EAU guidelines on primary urethral carcinoma. Eur Urol 2013;64:823830.

5 Pizzocaro G, Algaba F, Horenblas S, et al: EAU penile cancer guidelines 2009. Eur Urol 2010;57:1002-1012.

-6 Kulkarni S, Barbagli G, Kirpekar D, Mirri F, Lazzeri M: Lichen sclerosus of the male genitalia and urethra: surgical options and results in a multicenter international experience with 215 patients. Eur Urol 2009;55:945-956. 
Lumen et al:: Revision of Perineal Urethrostomy Using a Meshed Split-Thickness Skin Graft

7 Schreiter F, Noll F: Mesh graft urethroplasty using split thickness skin graft or foreskin. J Urol 1989;142:1223-1226.

8 Blandy JP, Singh M, Tresidder GC: Urethroplasty by scrotal flap for long urethral strictures. Br J Urol 1968;40:261-267.

-9 Kamat N: Perineal urethrostomy stenosis repair with buccal mucosa: description of technique and report of four cases. Urology 2008;72:1153-1155.

10 Johanson B: The reconstruction in stenosis of the male urethra (in German). Z Urol 1953;46:361-375.

11 Lumen N: Monti's procedure as an alternative technique in complex urethral distraction defect. Editorial comment. Int Braz J Urol 2010;36:324-325.

12 Dalpiaz 0, Kerschbaumer A, Pelzer A, et al: Single-stage dorsal inlay split-skin graft for salvage anterior urethral reconstruction. BJU Int 2008;101:1565-1570.

13 Carr LK, MacDiarmid SA, Webster GD: Treatment of complex anterior urethral stricture disease with mesh graft urethroplasty. J Urol 1997;157:104-108.

14 Pfalzgraf D, Olianas R, Schreiter F, Fisch M: Two-staged urethroplasty: buccal mucosa and mesh graft techniques. Aktuelle Urol 2010;41(suppl 1):S5-S9.

15 Kessler TM, Schreiter F, Kralidis G, Heitz M, Olianas R, Fisch M: Long-term results of surgery for urethral stricture: a statistical analysis. J Urol 2003;170:840-844.
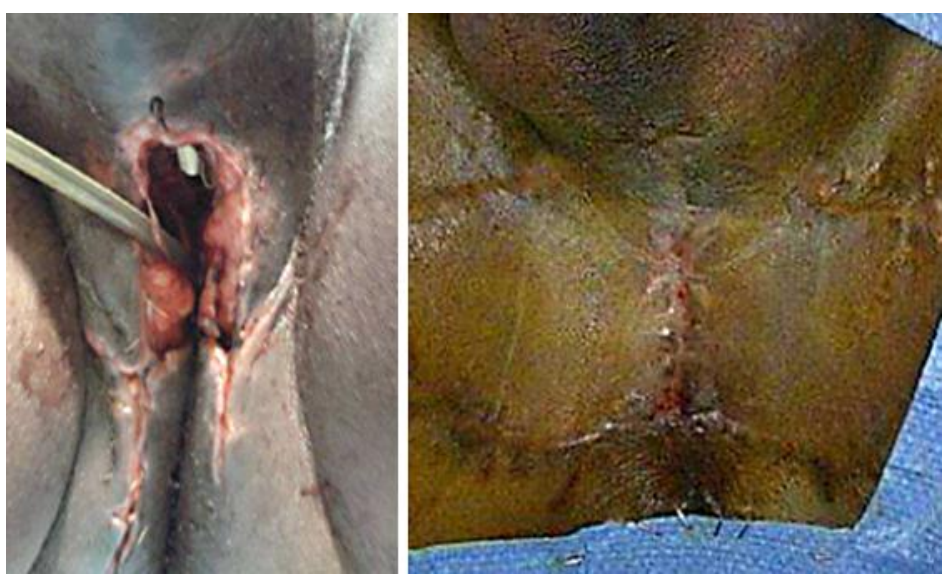

Fig. 1. Status 1 week after Blandy perineostomy, complicated by postoperative bleeding and wound dehiscence (left). Status 4 months after Blandy perineostomy and secondary wound healing. The perineal urethrostomy is completely obliterated (right). 
Case Reports in

Nephrology and

Urology
Case Rep Nephrol Urol 2014;4:12-17

DOI: $10.1159 / 000358556$

umen et al.: Revision of Perineal Urethrostomy Using a Meshed Split-Thickness Skin Graft

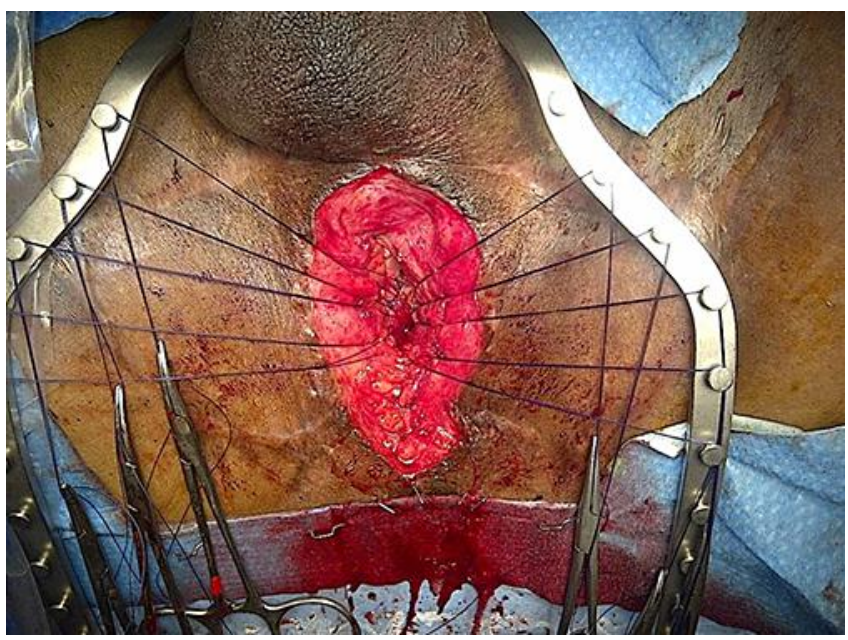

Fig. 2. The urethra has been opened and surrounding fibrotic tissue has been resected. Vicryl 4.0 sutures are placed to prepare the anastomosis for the graft.



Fig. 3. Immediate postoperative view (left) and after 6 weeks (right). 